\title{
Amiloidosis extrema asociada a mieloma múltiple e insuficiencia renal terminal. Caso clínico
}

\author{
CAMILA PEÑA O. ${ }^{a}$, MARVILA INTRIAGO B. ${ }^{a}$, \\ ANA MARÍA GRAY H., MARÍA ELENA CABRERA C.

\section{Primary amyloidosis associated with multiple myeloma and renal failure. Case report}

We report a 53-year-old female on chronic dialysis, presenting with weight loss, the development of big soft masses in the shoulders ("shoulder pads"), forearms and buttocks, macroglossia and rigidity of hands and lower limbs. Laboratory confirmed the presence of amyloidosis and myeloma with lambda chain restricted plasma cell infiltration of bone marrow. The diagnosis of multiple myeloma associated with primary amyloidosis in a patient with terminal renal failure, was reached.

(Rev Med Chile 2011; 139: 1471-1474).

Key words: Amyloidosis; Multiple myeloma; Renal insufficiency, chronic.

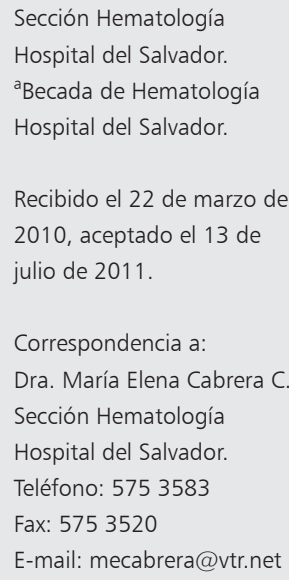

L a amiloidosis primaria (AL) es una enfermedad infrecuente causada por una neoplasia de células plasmáticas o más raramente una neoplasia linfoplasmocitaria. Las células neoplásicas secretan cadenas livianas de inmunoglobulina intactas o fragmentos, que cambian su conformación, se agregan y forman fibrillas que se depositan en varios tejidos, causando daño orgánico ${ }^{1}$. La incidencia es muy baja, 8 personas/millón de habitantes/año. En Chile existe poca información, sólo comunicación de casos ${ }^{2}$. Aproximadamente 5-15\% de pacientes con mieloma múltiple (MM) desarrollan amiloidosis. Según una serie de la Clínica Mayo, la frecuencia depende del componente M: IgA 2\%, IgG 5\%, cadenas livianas 17\% e IgD 19\% ${ }^{3}$.

La clínica es polimorfa y puede manifestarse por compromiso cardiaco, renal, sistema nervioso periférico, gastrointestinal, osteoarticular o cutáneo. La pérdida de peso puede ser severa en etapas terminales ${ }^{4}$. La cadena ligera lambda es la más frecuentemente involucrada (80\%). La sobrevida es de alrededor de 2 años, pero más corta, si se asocia a mieloma. Las alternativas terapéuticas incluyen desde quimioterapia, inmunomoduladores a trasplante de progenitores hematopoyéticos.
Se presenta el caso clínico de una paciente que se mantuvo en hemodiálisis crónica durante un año, sin diagnóstico etiológico del daño renal, a quien se diagnosticó mieloma y amiloidosis. El objetivo fue demostrar la semiología de amiloidosis extrema, situación muy infrecuente.

\section{Caso clínico}

Mujer de 53 años, domiciliada en Calama, con diagnóstico de insuficiencia renal terminal de causa desconocida, en programa de hemodiálisis hacía 1 año. Durante este período se agregó baja de peso de $30 \mathrm{~kg}$, cefalea, dolor polineuropático en guante y en calcetín y poliartralgias. En los últimos meses, había aparecido aumento de volumen blando, indoloro, progresivo, en ambos hombros, antebrazo derecho y ambos muslos, inmovilidad articular de ambas manos y rodillas, postración y diarrea las últimas dos semanas. Fue derivada a nuestro hospital para estudio de posible enfermedad reumatológica. Al examen físico se evidenció paciente caquéctica, pálida, postrada. Destacó macroglosia con lengua geográfica (Figura 1), masas 


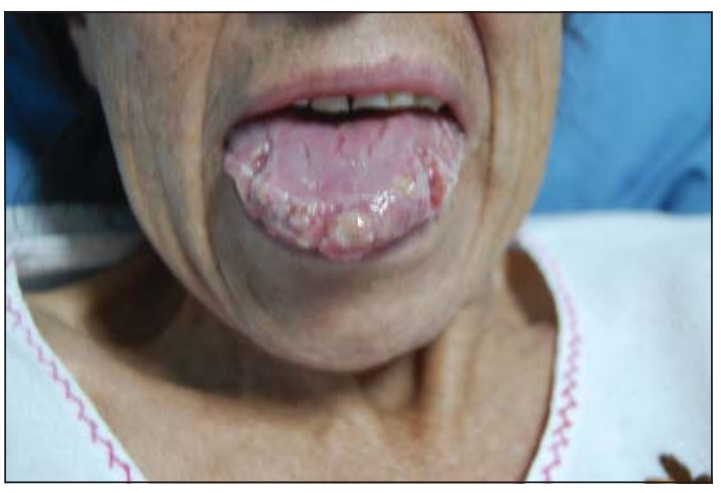

Figura 1. Macroglosia.

gomosas en ambos hombros (periarticulares) de 6 $\mathrm{cm}$ : signo de shoulder pad (Figuras $2 \mathrm{a}$ y $2 \mathrm{~b}$ ), masa de $3 \mathrm{~cm}$ en antebrazo derecho y $12 \mathrm{~cm}$ en ambos muslos, manos contracturadas en flexión y rigidez de ambas rodillas. Los exámenes de laboratorio mostraron hemoglobina $8,7 \mathrm{~g} / \mathrm{dL}$, creatinina 6,5 $\mathrm{mg} / \mathrm{dL}$, calcemia $12 \mathrm{mg} / \mathrm{dL}$, albúmina $1,9 \mathrm{~g} / \mathrm{L}$, proteína total 5,4 g/dL y triglicéridos $450 \mathrm{mg} / \mathrm{dL}$. Debido a la fuerte sospecha de mieloma múltiple y amiloidosis asociada, se realizó mielograma que mostró infiltrado de $25 \%$ de células plasmáticas. El inmunofenotipo de médula ósea mostró presencia de células plasmáticas con restricción de cadena liviana lambda y diagnóstico de mieloma. La electroforesis de proteínas mostró un pequeño componente $\mathrm{M}$ en G1 y cuantificación de inmunoglobulinas muy disminuidas, IgA $27,7 \mathrm{mg} / \mathrm{dL}$, IgG e IgM de $8,8 \mathrm{mg} / \mathrm{dL}$. No fue posible estudiar proteinuria ya que la paciente no presentaba diuresis. La biopsia de masa de muslo izquierdo, informó tejido fibroadiposo, escaso tejido muscular estriado y depósitos positivos para Rojo Congo, confirmando el diagnóstico de amiloidosis. Las radiografías óseas mostraron lesiones líticas en cadera y fémur derecho y aumento de partes blandas en correspondencia a masas descritas anteriormente, sin relación con estructuras óseas. Una electromiografía mostró una neuropatía por atrapamiento de nervio peroneo y nervio mediano derecho. Se realizó un TAC de tórax, abdomen y pelvis, que mostró depósitos de partes blandas en hombros (periarticulares), pericardio, pared intestinal, hepatomegalia y aumento de volumen de cabeza de páncreas. No se realizó ecocardiograma. Se concluyó que se trataba de mieloma múltiple,

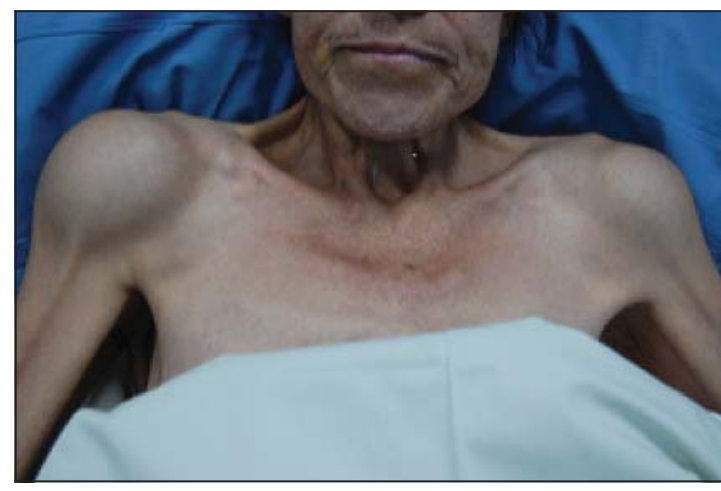

Figura 2a. Signo de "shoulder pad" u hombrera.

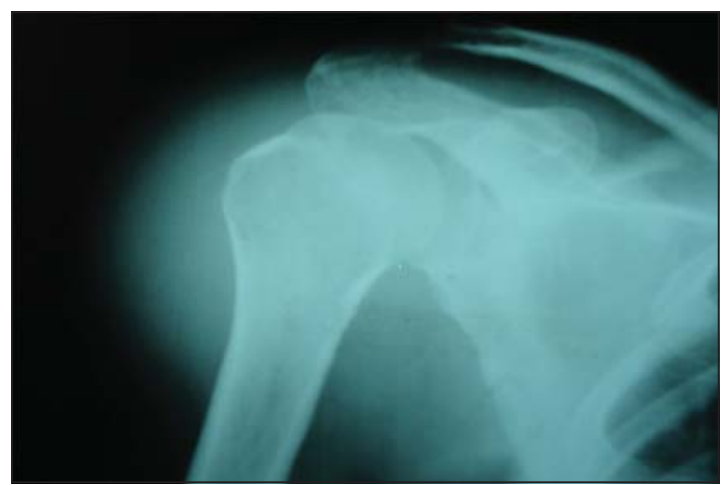

Figura $\mathbf{2 b}$. Radiografía hombro derecho que muestra el aumento de partes blandas periarticular.

amiloidosis asociada e insuficiencia renal terminal. Recibió bisfosfonatos, con reducción de la hipercalcemia y se inició tratamiento con melfalan 10 $\mathrm{mg} /$ día y prednisona $40 \mathrm{mg}$ /día por 4 días. Luego se agregó talidomida $50 \mathrm{mg} /$ día. Lamentablemente, la paciente falleció al mes de tratamiento.

\section{Discusión}

La amiloidosis AL asociada a mieloma múltiple (MM) se presenta con síntomas dependientes de los órganos comprometidos. En la paciente descrita, se observaron manifestaciones clínicas frecuentes y otras menos comunes pero llamativas.

La macroglosia con indentaciones laterales es una manifestación fácilmente identificable, presente en $12-40 \%$ de los pacientes ${ }^{5}$ y evidente en nuestro caso (Figura 1). El compromiso 
del sistema nervioso periférico, con neuropatía compresiva sensitiva como el síndrome de túnel carpiano o autonómica ${ }^{6}$ es común. Nuestra paciente presentaba dolor neuropático característico en guante y calcetín y la electromiografía mostró compresión de nervios periféricos. La diarrea por síndrome de malabsorción ${ }^{7}$ que presentó en las últimas semanas, podría atribuirse a neuropatía del sistema nervioso autónomo.

Los síntomas cardiacos pueden ser de tipo conductivo como bloqueos o alteraciones del ritmo cardiaco o de tipo restrictivo que llevan a insuficiencia cardiaca y muerte en aproximadamente $40 \%$ de los pacientes ${ }^{8}$. Este daño no fue demostrado en nuestro caso, ya que no se realizó ecocardiograma.

Las manifestaciones más prominentes fueron osteoarticulares, poco frecuentes en amiloidosis. De hecho, la paciente fue derivada con diagnóstico de probable enfermedad reumatológica por artralgias y rigidez de manos y rodillas. El depósito de amiloide en la sinovial produce una hipertrofia nodular del sinovio y edema de los tejidos blandos secundario, produciendo una pseudohipertrofia articular y el signo característico del "shoulder pad" u hombrera, como se observó en forma tan evidente en nuestra paciente (Figura 2). La ubicación más frecuente es la articulación glenohumeral, resultando en este peculiar signo, patognomónico de amiloidosis de inmunoglobulinas ${ }^{9}$. Cuando la mano se encuentra comprometida puede observarse nodularidad y engrosamiento de la fascia palmar con contracturas en flexión y marcada debilidad. Nuestro caso presentaba contractura en flexión de ambas manos y rodillas, con notable dificultad para extender las extremidades. Las manifestaciones renales pueden expresarse desde proteinuria asintomática hasta un síndrome nefrótico ${ }^{10}$. En nuestro caso no fue posible estudiar la orina, ya que la paciente no tenía diuresis. Sin embargo, es probable que el daño renal haya sido provocado por la eliminación de cadenas ligeras lambda.

El diagnóstico de amiloidosis AL se basa en la histología del órgano afectado y la tinción de Rojo Congo. La biopsia de tejido subcutáneo abdominal tiene sensibilidad de $57-85 \%$ y especificidad de $92-100 \%$ y la biopsia rectal sensibilidad de $84 \%{ }^{11}$.

Frente a un caso de amiloidosis, se debe investigar siempre la presencia de mieloma. En nuestro caso, éste se basó, según los criterios de la $\mathrm{OMS}^{12}$, en la presencia de más de 10\% de células plasmáticas clonales en la médula ósea y la presencia de daño orgánico: hipercalcemia, insuficiencia renal, anemia y lesiones osteolíticas. Si bien no se demostró una proteína $\mathrm{M}$ en el suero, en una paciente en anuria, la electroforesis de proteínas mostró un pequeño componente $\mathrm{M}$ y una disminución severa de las inmunoglobulinas normales, hecho característico del mieloma sintomático avanzado.

La insuficiencia renal de la paciente es multifactorial. Los hechos ciertos son que existe amiloidosis y mieloma, por lo tanto, la etiología más probable es la asociación de ambas enfermedades, como una unidad diagnóstica.

El tratamiento para la amiloidosis AL consiste en erradicar la célula plasmática clonal que produce la cadena liviana amiloidogénica. Para ello se ha propuesto utilizar altas dosis de melfalán y trasplante autólogo de progenitores hematopoyéticos (TPH), con respuesta hematológica completa en $25-50 \%{ }^{13,14}$. La enfermedad renal y/o hepática avanzada contraindicaría la terapia y el inconveniente es la alta toxicidad, con mortalidad de 12$14 \%$, mayor cuando existe compromiso cardiaco.

El tratamiento clásico con melfalán-prednisona ${ }^{15}$ confiere una sobrevida media sólo de 12 meses. El uso de dosis altas de dexametasona es menos tóxico, con una esperanza de vida de 2,6 años en la mitad de los pacientes ${ }^{16}$. También se ha usado esquemas como VAD (vincristina, doxorubicina, dexametasona) o C-VAD (ciclofosfamida más VAD) en formas agresivas. Otras alternativas son el uso de talidomida o lenalidomida, asociadas a corticoides ${ }^{17}$.

En resumen, presentamos un caso de mieloma y amiloidosis extrema, por las características docentes del caso. Quizás, un tratamiento más precoz del mieloma hubiera evitado la acumulación tan masiva de amiloide que observamos en nuestra paciente.

\section{Referencias}

1. Merlini G, Bellotti V. Molecular mechanisms of amyloidosis. N Engl J Med 2003; 349: 583-96.

2. Palma CL, Grunholz D, Osorio G. Amiloidosis. Comunicación de 11 casos y revisión de la literatura. Rev Med Chile 2005; 133: 655-61.

3. Bladé J, Lust JA, Kyle RA. Immunoglobulin D myeloma: presenting features, response to therapy, and survival in 
a series of 53 patients. J Clin Oncol 1994; 12: 2398-404.

4. Vega J, Martínez M, Sánchez P, Sales J, Rodríguez M. Manifestaciones cutáneas de la amiloidosis sistémica asociada a mieloma, Med Cutan Lat Am 2004; 32: 211-4.

5. Moroni A, Benavides A, Retamal Y. Macroglosia y amiloidosis oculta. Rev Med Chile 2002; 130: 215-8.

6. Kang EH, Lee EB, Im CH, Kim CJ, Park JJ, Lee JC, et al. A case of femoral compressive neuropathy in AL amyloidosis, J Korean Med Sci 2005; 20: 524-7.

7. Brunt E, Tiniakos D. Metabolic storage diseases: amyloidosis. Clin Liver Dis 2004; 8: 915-30.

8. Masuda I, Pereiro G. Pasca J, Lastiri H. Miocardiopatía restrictiva. Amiloidosis. Algoritmo diagnóstico. Rev Fed Arg Cardiol 2006; 35: 88-91.

9. Guerreiro de Moura CG. Images in clinical medicine. "Shoulder Pad" Sign. N Engl J Med 2004; 351; 25: e23.

10. Bergesio F, Ciciani AM, Manganaro M, Palladini G, Santostefano M. Renal involvement in systemic amyloidosis. Nephrol Dial Transplant 2008; 23: 941-51.

11. Van Gameren II, Hazenberg BP, Bijzet J, van Rijswijk MH. Diagnostic accuracy of subcutaneous abdominal fat tissue aspiration for detecting systemic amyloidosis and its utility in clinical practice. Arthritis Rheum 2006; 54: 2015-21.

12. Swerdlow SH, Campo E, Harris NL, Jaffe ES, Pileri SA,
Stein $\mathrm{H}$, et al. WHO classification of tumours of Haematopoietic and Lymphoid tissues. IARC Press, Lyon, France, 2008.

13. Comenzo RL. How I treat amyloidosis. Blood 2009; 114: 3147-57.

14. Gertz MA, Lacy MQ, Dispenzieri A, Hayman SR, Kumar S. Transplantation for amyloidosis. Curr Opin Oncol 2007; 19: 136-41

15. Palladini G, Pefetti V, Obici L, Caccialanza R, Semino A, Adami F, et al. Association of melphalan and high-dose dexamethasone is effective and well tolerated in patients with AL (primary) amyloidosis who are ineligible for stem cell transplantation. Blood 2004; 103: 2936-8.

16. Dhodapkar MV, Hussein MA, Rasmussen E, Solomon A, Larson RA, Crowley JJ, et al. Clinical efficacy of highdose dexamethasone with maintenance dexamethasone/ alpha interferon in patients with primary systemic amyloidosis: results of United States Intergroup Trial Southwest Oncology Group (SWOG) S9628. Blood 2004; 104: 3520-6.

17. Palladini G, Perfetti V, Perlini S, Obici L, Lavatelli F, Caccialanza $\mathrm{R}$, et al. The combination of thalidomide and intermediate-dose dexamethasone is an effective but toxic treatment for patients with primary amyloidosis (AL). Blood 2005; 105: 2949-51. 